\title{
ON THE CALCULATION OF THE UNITS OF ALGEBRAIC NUMBER FIELDS
}

\author{
TAKASHI AZUHATA
}

\section{§. Introduction}

The method to find a system of fundamental units were given by G. F. Voronoi in the case of purely cubic fields (see B. N. Delone and D. K. Faddeev [1]), and by K. K. Billebic [2] in general case of algebraic number fields except for quadratic fields. But they are rather complicated for direct calculation. On the other hand, in some special cases, units can be calculated by the Jacobi-Perron algorithm which is a generalization of the continued fractional expansion (see, for example, L. Bernstein [3]). In this paper, we will show the new method to calculate a system of independent units by multiplying several numbers. We will give some examples for purely cubic fields in Section 3 .

\section{$\S 1$. Preliminaries}

It is known that the fundamental units of real quadratic fields are calculated by the continued fractional expansion. We reconsider this algorithm from the viewpoint of the sequence of ideals. Let $\omega$ be a reduced quadratic irrational with discriminant $d>0$, where $d$ is the discriminant of a real quadratic field $\boldsymbol{Q}(\sqrt{ } \bar{d})$, and let

$$
\omega_{0}=\omega, \quad \omega_{i}=k_{i}+1 / \omega_{i+1}, \quad k_{i}=\left[\omega_{i}\right] \quad(i \geq 0)
$$

be the continued fractional expansion of $\omega$. If we put $\omega_{i}=\left(\sqrt{ } d+b_{i}\right) / 2 a_{i}$ and $\mathfrak{U}_{i}=\left[a_{i},\left(\sqrt{d}+b_{i}\right) / 2\right]$ where $[\alpha, \beta]=Z \alpha+Z \beta$, then $\mathfrak{A}_{i}$ is an ideal of $\boldsymbol{Q}(\sqrt{ } \bar{d})$. We see that

$$
2 a_{i} k_{i}-b_{i}=b_{i+1}, \quad d-\left(2 a_{i} k_{i}-b_{i}\right)^{2}=4 a_{i} a_{i+1}
$$

from (1) by simple calculation. So we get

Received December 10, 1984. 


$$
\begin{aligned}
\frac{1}{2}\left(\sqrt{d}+b_{i+1}\right) \mathfrak{A}_{i} & =\frac{1}{2}\left(\sqrt{d}+b_{i+1}\right)\left[a_{i}, \frac{1}{2}\left(\sqrt{d}+b_{i}-2 a_{i} k_{i}\right)\right] \\
& =a_{i} \mathfrak{A}_{i+1}=N\left(\mathfrak{U}_{i}\right) \mathfrak{Y}_{i+1} .
\end{aligned}
$$

We notice that $\left(\sqrt{d}+b_{i+1}\right) / 2$ is an element of $\mathfrak{Y}_{i}^{\prime}$ which is a conjugate of $\mathfrak{U}_{i}$. If the period of $\omega$ is $m$, then $\omega_{m}=\omega_{0}$ and $\mathfrak{U}_{m}=\mathfrak{U}_{0}$. Therefore we have

$$
\left(\prod_{i=1}^{m} \frac{1}{2}\left(\sqrt{ } \bar{d}+b_{i}\right)\right) \mathfrak{Y}_{0}=\left(\prod_{i=0}^{m-1} a_{i}\right) \mathfrak{Y}_{0}
$$

Hence $\varepsilon=\omega_{0} \omega_{1} \cdots \omega_{m-1}$ is a unit of $\boldsymbol{Q}(\sqrt{d})$. As a matter of fact, $\varepsilon$ is a fundamental unit.

\section{§2. General method}

Let $K$ be an algebraic number field of degree $n \geq 3$ with discriminant $D$, and $\sigma_{i}: K \rightarrow C$ be the embeddings of $K$ into the field of complex numbers. As usual, we assume that $\sigma_{1}(K), \cdots, \sigma_{r_{1}}(K)$ are real fields, $\sigma_{r_{1+1}}(K), \cdots, \sigma_{n}(K)$ are complex fields and $\sigma_{r_{1}+r_{2}+i}(K)$ is a complex conjugate of $\sigma_{r_{1}+i}(K)\left(1 \leq i \leq r_{2}\right)$ with $r_{1}+2 r_{2}=n$. We put $N=N_{K / \boldsymbol{Q}}, \quad \xi^{(i)}=\sigma_{i}(\xi)$ and $N^{\prime}(\xi)=N(\xi) / \xi$ for $\xi \in K^{\times}$. For any fixed $\ell$ with $1 \leq \ell \leq r_{1}+r_{2}$, we now construct the unit $\varepsilon_{\ell}$ of $K$ satisfying

$$
\left|\varepsilon_{\ell}^{(\ell)}\right|>1, \quad\left|\varepsilon_{\ell}^{(k)}\right|<1 \quad\left(1 \leq k \leq r_{1}+r_{2}, k \neq \ell\right) .
$$

Then, it is well-known that any $r$ units among $\varepsilon_{1}, \varepsilon_{2}, \cdots, \varepsilon_{r_{1}+r_{2}}$ construct a system of independent units where $r=r_{1}+r_{2}-1$. For this purpose, we first notice that for any integral ideal $\mathfrak{A}$ of $K$ and for any integer $\xi \neq 0$ in $\mathfrak{A}, N^{\prime}(\xi) \mathfrak{U}$ is an integral ideal of $K$, and is divisible by $N(\mathfrak{U})$. In fact, considering in certain Galois field $L$ containing $K$, any conjugate $\xi^{\sigma}$ of $\xi$ is an integer of $L$ and $N^{\prime}(\xi)=N(\xi) / \xi$ is in $K$. So $N^{\prime}(\xi)$ is an integer of $K$. By the same argument, $N^{\prime}(\mathfrak{X})=\mathfrak{A}^{-1} N(\mathfrak{U})$ is an integral ideal of $K$. Hence we get $N(\mathfrak{U}) \mid N^{\prime}(\xi) \mathfrak{U}$ from $\xi^{\sigma} \in \mathfrak{U}^{\sigma}$ and $N^{\prime}(\mathfrak{H}) \mid N^{\prime}(\xi)$. Then, we can construct the sequence of integral ideals $\mathfrak{U}_{0}, \mathfrak{U}_{1}, \cdots, \mathfrak{A}_{i}, \cdots$ and the sequence of integers $\xi_{0}, \xi_{1}, \cdots, \xi_{i}, \cdots$ of $K$ such that

$$
0 \neq \xi_{i} \in \mathfrak{A}_{i}, \quad N^{\prime}\left(\xi_{i}\right) \mathfrak{U}_{i}=N\left(\mathfrak{U}_{i}\right) \mathfrak{A}_{i+1} \quad(i \geq 0) .
$$

Moreover, by Minkowski's linear forms theorem, we can choose above $\xi_{i}$ so that the following conditions are also satisfied: 


$$
\begin{aligned}
& \left|\xi_{i}^{(k)}\right|<N\left(\mathfrak{U}_{i}\right)^{1 /(n-1)} \quad\left(1 \leq k \leq r_{1}+r_{2}, k \neq \ell\right), \\
& \left|\xi_{i}^{(\ell)}\right|<\Delta \quad \text { if } \ell \leq r_{1}, \\
& \left|\xi_{i}^{(\ell)}\right|^{2}<\Delta N\left(\mathfrak{U}_{i}\right)^{1 /(n-1)} \quad \text { if } \ell>r_{1},
\end{aligned}
$$

where $\Delta$ is any real number greater than $\Delta_{0}=(2 / \pi)^{r_{2}}|D|^{1 / 2}$. It follows from

$$
N\left(\mathfrak{H}_{i}\right)^{n} N\left(\mathfrak{A}_{i+1}\right)=\left|N\left(\xi_{i}\right)\right|^{n-1} N\left(\mathfrak{U}_{i}\right)<\Delta^{n-1} N\left(\mathfrak{I}_{i}\right)^{n}
$$

that $N\left(\mathscr{U}_{i}\right)<\Delta^{n-1}(i \geq 1)$. If we take $\Delta$ sufficiently close to $\Delta_{0}$, then we get $N\left(\mathfrak{H}_{i}\right) \leq \Delta_{0}^{n-1}(i \geq 1)$ since $N\left(\mathfrak{U}_{i}\right)$ is a rational integer. There are only finite integral ideals $\mathfrak{U}$ with $N(\mathfrak{U}) \leq \Delta_{0}^{n-1}$ in $K$. So we have $\mathfrak{A}_{s}=\mathfrak{A}_{t}$ for some positive integers $s, t$ with $s<t$. Then, we have the following

TheOREM 1. $\varepsilon_{\ell}=\prod_{i=s}^{t-1} \xi_{i} N\left(\mathfrak{H}_{i}\right) / N\left(\xi_{i}\right)$ is a unit satisfying (2).

Proof. It follows from (3) and $\mathfrak{A}_{s}=\mathfrak{A}_{t}$ that

$$
\left(\prod_{i=s}^{t-1} N^{\prime}\left(\xi_{i}\right)\right) \mathfrak{H}_{s}=\left(\prod_{i=s}^{t-1} N\left(\mathfrak{H}_{i}\right)\right) \mathfrak{U}_{s} .
$$

Therefore

$$
\varepsilon_{\ell}=\prod_{i=s}^{t-1} \xi_{i} N\left(\mathfrak{U}_{i}\right) / N\left(\xi_{i}\right)=\prod_{i=s}^{t-1} N\left(\mathfrak{H}_{i}\right) / N^{\prime}\left(\xi_{i}\right)
$$

is a unit. We notice that $\prod\left|N\left(\xi_{i}\right)\right|=\prod N\left(\mathfrak{H}_{i}\right)^{n /(n-1)}$ since $\left|\prod N\left(\mathscr{U}_{i}\right)^{n} / N\left(\xi_{i}\right)^{n-1}\right|$ $=1$. Hence we have (2) from

$$
\left|\varepsilon_{\ell}^{(k)}\right|=\prod\left|\xi_{i}^{(k)} N\left(\mathfrak{H}_{i}\right) / N\left(\xi_{i}\right)\right|=\prod\left|\xi_{i}^{(k)}\right| / N\left(\mathfrak{H}_{i}\right)^{1 /(n-1)}<1 .
$$

This completes the proof.

We note that $\left(\prod_{i=s}^{k} \xi_{i} N\left(\mathfrak{U}_{i}\right)\right) /\left(\prod_{i=s}^{k-1} N\left(\xi_{i}\right)\right)(\mathrm{s}+1 \leq k \leq t-1)$ are integral since $N\left(\xi_{i}\right) \mid \xi_{i} N^{\prime}\left(\mathfrak{A}_{i}\right) \mathfrak{A}_{i+1}$. It is not easy to find the base of an ideal of $K$. But if the base of $\mathfrak{U}_{i}$ is given, then we can find easily the base of $\mathfrak{A}_{i+1}$ since $\alpha \mathfrak{U}=\left[\alpha \omega_{1}, \cdots, \alpha \omega_{n}\right]$ if $\mathfrak{Q}=\left[\omega_{1}, \cdots, \omega_{n}\right]=Z \omega_{1}+\cdots+Z \omega_{n}$. So, if we know the integral base $\left\{\alpha_{1}=1, \alpha_{2}, \cdots, \alpha_{n}\right\}$ of $K$ and put $\mathfrak{A}_{0}=O_{K}$, the integer ring of $K$, then the bases of ideals $\mathfrak{A}_{i}$ are explicitly calculated. Furthermore, we can take the base $\left\{\omega_{i 1}, \cdots, \omega_{i n}\right\}$ of $\mathfrak{A}_{i}$ as follows:

$$
\omega_{i k}=\sum_{j=1}^{k} a_{j k} \alpha_{j} \text { with } a_{j k} \in Z, a_{j j}>a_{j k} \geq 0 .
$$

Then $\mathfrak{A}_{i} \cap \boldsymbol{Z}=\left(a_{11}\right), N\left(\mathfrak{U}_{i}\right)=\prod a_{j j}$, and hence we can decide immediately whether $\mathfrak{A}_{s}=\mathfrak{A}_{t}$ or not.

Remark 1. The sequence of the ideals $\mathfrak{A}_{0}, \mathfrak{A}_{1}, \ldots, \mathfrak{A}_{i}, \ldots$ is not unique since the choice of $\xi_{i}$ satisfying (4) may be not unique. 


\section{§3. The case of purely cubic fields}

Let $K$ be a real cubic field with negative discriminant and $O_{K}$ be the integer ring of $K$. Then, the unit group of $K$ is generated by \pm 1 and the fundamental unit $\varepsilon>1$. We take the $\Delta$ sufficiently close to $\Delta_{0}$ so that $N\left(\mathfrak{U}_{i}\right) \leq \Delta_{0}^{2}(i \geq 1)$. Now we consider the sufficient condition for the $\varepsilon_{1}$ to be the fundamental unit when $\varepsilon>\Delta$. Every principal ideal $\mathfrak{A}$ of $O_{K}$ may be written as $\mathfrak{A}=(\alpha)$ with $1 \leq \alpha<\varepsilon$. Let $P$ be the set of all principal ideals $\mathfrak{A}=(\alpha)$ with $\alpha \in O_{K}, 1 \leq \alpha<\Delta, N(\mathfrak{U}) \leq \Delta_{0}^{2}$ and let $\mathfrak{U}_{0}=O_{K}$, $\mathfrak{A}_{1}, \cdots, \mathfrak{U}_{m-1}, \mathfrak{A}_{m}=O_{K}$ be the sequence of ideals of $K$ satisfying the conditions (3) and (4) with $\ell=1$. If we put $\alpha_{i}=\prod_{k=i}^{m-1} \xi_{k} N\left(\mathfrak{U}_{k}\right) / N\left(\xi_{k}\right)$, then $\alpha_{i} \in O_{K}, \alpha_{0}>\alpha_{1}>\cdots>\alpha_{m-1}>1, \mathfrak{A}_{i}=\left(\alpha_{i}\right)$ and $\varepsilon_{1}=\alpha_{0}$ is a unit. Then, we have the following

TheOREm 2. If none of the ideals $\mathfrak{A}_{1}, \mathfrak{A}_{2}, \cdots, \mathfrak{A}_{u}$ are in $P$ with $u$ satisfying $\alpha_{u}>\sqrt{\varepsilon_{1}} \geq \alpha_{u+1}$, then $\varepsilon_{1}$ is the fundamental unit.

Proof. Assume that $\varepsilon_{1}=\varepsilon^{e}$ with $e>1$. Then we have $\alpha_{v}>\varepsilon_{1} \varepsilon^{-1} \geq$ $\alpha_{v+1}$ with $v \leq u$ and $\mathfrak{U}_{v}=\left(\alpha_{v}\right)=\left(\alpha_{v} \varepsilon \varepsilon_{1}^{-1}\right)$. It follows from

$$
\alpha_{v} \varepsilon \varepsilon_{1}^{-1} \leq \alpha_{v} / \alpha_{v+1}=\xi_{v} N\left(\mathscr{A}_{v}\right) / N\left(\xi_{v}\right) \leq\left|\xi_{v}\right|<\Delta
$$

that $\mathfrak{U}_{v} \in P$, which is a contradiction. This proves our assertion.

Remark 2. The similar argument applies to the case of totally imaginary quartic fields.

In particular, if $K=\boldsymbol{Q}(\sqrt[3]{d})$ where $d=m n^{2}$ with square-free integers $m>n \geq 1,(m, n)=1, m^{2} \neq n^{2}(\bmod 9)$, then the integral base of $K$ is given by $1, \theta=\sqrt[3]{d}, \tilde{\theta}=\theta^{2} / n$ and the discriminant of $K$ is $D=-27 m^{2} n^{2}$. Let $\mathfrak{I}_{i}=\left[a, b+b_{1} \theta, c+c_{1} \theta+c_{2} \tilde{\theta}\right]$ be an ideal of $K$ and

$$
\begin{aligned}
f_{j} & =a x+\left(b+b_{1} \theta^{(j)}\right) y+\left(c+c_{1} \theta^{(j)}+c_{2} \tilde{\theta}^{(j)}\right) z \quad(j=1,2,3), \\
\tilde{D} & =\left[\begin{array}{ccc}
1 & 1 & 0 \\
\theta & -\theta / 2 & \sqrt{3} \tilde{\theta} / 2 \\
\tilde{\theta} & -\tilde{\theta} / 2 & -\sqrt{3} \tilde{\theta} / 2
\end{array}\right], \quad A=\left[\begin{array}{lll}
a & 0 & 0 \\
b & b_{1} & 0 \\
c & c_{1} & c_{2}
\end{array}\right], \\
\Delta & >6 \sqrt{3} m n / \pi, \quad \beta=\left(a b_{1} c_{1}\right)^{1 / 2} .
\end{aligned}
$$

It follows from the inequalities $\left|f_{1}\right|<\Delta,\left|f_{2}\right|=\left|f_{3}\right|<\beta$ that $(x, y, z) A \tilde{D}=$ $(\lambda, \mu, \nu)$ with real numbers $\lambda, \mu, \nu$ such that $|\lambda|<\Delta, \mu^{2}+\nu^{2}<\beta^{2}$. Denote by $\mathbf{b}_{1}, \mathbf{b}_{2}, \mathbf{b}_{3}$ the row vectors of $\tilde{D}^{-1} A^{-1}$, then $(x, y, z)$ is a point in the parallelotope 


$$
V=\left\{\lambda \mathbf{b}_{1}+\mu \mathbf{b}_{2}+\nu \mathbf{b}_{3}|| \lambda|<\Delta,| \mu|<\beta,| \nu \mid<\beta\right\} .
$$

Hence we may choose $\xi_{i}$ from a suitable non-zero lattice point in $V$.

Examples. Denote by $\left[a ; b, b_{1} ; c, c_{1}, c_{2}\right]$ an ideal $\left[a, b+b_{1} \theta, c+c_{1} \theta\right.$ $\left.+c_{2} \tilde{\theta}\right]$ and put $\gamma_{i}=\xi_{i} N\left(\mathfrak{U}_{i}\right) / N\left(\xi_{i}\right)$.

(i) $d=5$,

$$
\begin{array}{ll}
\mathfrak{U}_{0}=[1 ; 0,1 ; 0,0,1], & \gamma_{0}=(3+2 \theta+\tilde{\theta}) / 2, \\
\mathfrak{A}_{1}=[2 ; 0,2 ; 1,1,1], & \gamma_{1}=(1+\theta+\tilde{\theta}) / 4, \\
\mathfrak{A}_{2}=[4 ; 2,2 ; 2,0,2], & \gamma_{2}=(2+2 \theta) / 3, \\
\mathfrak{I}_{3}=[3 ; 0,3 ; 1,2,1], & \gamma_{3}=4+2 \theta+\tilde{\theta}, \\
\mathfrak{A}_{4}=\mathfrak{A}_{0}, \quad \varepsilon=\gamma_{0} \gamma_{1} \gamma_{2} \gamma_{3}=41+24 \theta+14 \tilde{\theta},
\end{array}
$$

(ii) $d=11, \quad \mathfrak{X}_{0}=[1 ; 0,1 ; 0,0,1], \quad \gamma_{0}=(4+2 \theta+\tilde{\theta}) / 9$,

$$
\begin{array}{ll}
\mathfrak{I}_{1}=[9 ; 3,3 ; 6,0,3], & \gamma_{1}=(3+3 \theta) / 4, \\
\mathfrak{I}_{2}=[4 ; 0,4 ; 1,3,1], & \gamma_{2}=(5+3 \theta+\tilde{\theta}) / 3,
\end{array}
$$$$
\mathfrak{A}_{3}=[3 ; 0,3 ; 1,2,1], \quad \gamma_{3}=4+2 \theta+\tilde{\theta} \text {, }
$$$$
\mathfrak{A}_{4}=\mathfrak{A}_{0}, \quad \varepsilon=\gamma_{0} \gamma_{1} \gamma_{2} \gamma_{3}=89+40 \theta+18 \tilde{\theta} \text {. }
$$

\section{REFERENCES}

[1] B. N. Delone and D. K. Faddeev, The theory of irrationalities of the third degree, Trudy Mat. Inst. Steklov, (1940), Trans. Amer. Math. Soc., 10 (1955).

[2] K. K. Billebic, On the units of algebraic fields of third and fourth degree, Mat. Sb., (1956) 123-136.

[ 3 ] L. Bernstein, The Jacobi-Perron algorithm, its theory and application, Lecture Note in Math., Springer, 207 (1971).

Department of Mathematics

Science University of Tokyo

26 Wakamiya, Shinjuku-ku

Tokyo, Japan 